\title{
Review of: "Health human resources challenges during COVID-19 pandemic; evidence of a qualitative study in a developing country"
}

ali sahebi

Potential competing interests: The author(s) declared that no potential competing interests exist.

Thank you for the opportunity to review this paper on a significant topic. Reviewing the article, I found it a high-quality study and I approve its publication provided that the three following points are taken into consideration:

1.Emphasize the need to perform this study and lack of knowledge in this area.

Furthermore, what is the novelty of this study?

2.The date of starting and terminating the study has not been mentioned.

3. The study limitations have not been mentioned. 